\title{
Optimización de parámetros del proceso de elaboración de chicha de jora
}

Optimization of parameters in the process of elaboration of chicha de jora

Recibido: diciembre 12 de 2017 | Revisado: febrero 22 de 2018 | Aceptado: abril 20 de 2018

\author{
Silvia Ara Rojas* \\ Ana Hurtado Alendes*2 \\ EdDY BARNETT MENDOZA ${ }^{3}$ \\ Luis Celi SaAvedra ${ }^{4}$ \\ Mónica Ramos Escuderos
}

\begin{abstract}
RESUMEN
Se evaluaron diferentes procesos de elaboración de chicha de jora para optimizar los principales parámetros fisicoquímicos y microbiológicos para obtener un producto que cumpla con los estándares de calidad para su consumo. Se utilizó como materia prima maíz jora comercial y dos tipos de maíz híbrido jaspeado rojo y negro, germinados bajo condiciones de laboratorio. Los mostos fueron obtenidos por litro de preparación con $200 \mathrm{~g}$ de maíz jora molido, $50 \mathrm{~g}$ de chancaca y $50 \mathrm{~g}$ de azúcar morena y fermentados usando como $0,5 \mathrm{~g}$ de levadura $S$. cereviciae, previamente activada. Durante cada fermentación se evaluaron: temperatura de fermentación, densidad, pH, grados Brix y grado alcohólico. Finalizada la fermentación, la chicha se filtró, envasó, pasteurizó a $80^{\circ} \mathrm{C}$ por $5 \mathrm{~min}$, selló al vacío y conservó a temperatura ambiente hasta su evaluación sensorial. A cada producto obtenido, le fue asignada su calidad microbiológica mediante métodos estandarizados. Sensorialmente, cuatro muestras de chicha de jora obtenidas bajo iguales condiciones de fermentación y una muestra adquirida comercialmente como control, fueron evaluadas por un panel de 13 jueces semientrenados mediante una prueba escalar de control de cinco puntos de intensidad creciente. La muestra de mayor preferencia fue evaluada por un panel de siete jueces entrenados para los perfiles de sabor, olor y textura/consistencia. Se estableció que se genera un buen producto y de gran aceptación (chicha de $\mathrm{pH} 4$ a 4,3 y 4 a $5^{\circ} \mathrm{OH}$ ) empleando $15,63^{\circ}$ Brix, $\mathrm{pH} 5,5$ y densidad entre 1,15 y $1,35 \mathrm{~g} / \mathrm{L}$, obtenidos de maíz jora germinado bajo condiciones controladas, con la adición de $100 \mathrm{~g} / \mathrm{L}$ de chancaca y $50 \mathrm{~g} / \mathrm{L}$ de azúcar morena y fermentados de 60 a 72 horas.
\end{abstract}

Palabras clave: chicha de jora, fermentación, optimización
Universidad de San Martín de Porres

*Autores principales

1 sarar@usmp.pe

2 ahurtado@usmp.pe

3 ebarnettm@usmp.pe

4 lcelis@usmp.pe

5 mramose@usmp.pe
Abstract

Different chicha de jora processes were evaluated to optimize the main physicochemical and microbiological parameters to obtain a product that meets the quality standards for its consumption. Commercial raw corn and two types of red and black variegated hybrid maize, germinated under laboratory conditions were used as a raw material. The musts were obtained per liter of preparation with $200 \mathrm{~g}$ of ground corn, $50 \mathrm{~g}$ of chancaca and $50 \mathrm{~g}$ of brown sugar and fermented using $0.5 \mathrm{~g}$ of yeast $\mathrm{S}$. cereviciae, previously activated. During each 
fermentation, values of fermentation temperature, density, $\mathrm{pH}$, Brix degrees and alcohol content were evaluated. After fermentation, the chicha was filtered, bottled, pasteurized at $80^{\circ} \mathrm{C}$ for $5 \mathrm{~min}$, sealed under vacuum and kept at room temperature until sensory evaluation. For each of the products obtained, its microbiological quality was assigned by standardized methods. Sensorially, four samples of chicha de jora obtained under the same conditions of fermentation and a sample acquired commercially as control, were evaluated by a panel of 13 semi-trained judges by means of a scalar control test of five points of increasing intensity. The most preferred sample was evaluated by a panel of seven trained judges checking its taste, odor and texture / consistency profiles. It was established that a good and highly accepted product is generated (chicha of $\mathrm{pH} 4$ to 4.3 and 4 to $5^{\circ} \mathrm{OH}$ ) using $15.63^{\circ}$ Brix, $\mathrm{pH} 5.5$ and density between 1.15 and $1.35 \mathrm{~g} / \mathrm{L}$, obtained from corn germinated under controlled conditions, with the addition of $100 \mathrm{~g} / \mathrm{L}$ of chancaca and $50 \mathrm{~g}$ / L of brown sugar and fermented from 60 to 72 hours.

Key words: chicha de jora, fermentation, optimization

\section{Introducción}

La chicha de jora es una bebida alcohólica, originaria del Perú, que se obtiene por la fermentación de la materia azucarada contenida en el mosto de malta del maíz. Se elabora desde el tiempo de los incas y su consumo fue común en todo el imperio incaico y hasta la actualidad se sigue elaborando en nuestro país y en otros de América del Sur como Ecuador, Colombia y Chile. Es una bebida que perdura en el tiempo. Se consume en las regiones de la sierra, de manera especial durante las festividades regionales, aunque con el pasar de los ańos y por diversos motivos como la inclusión de alimentos (bebidas) poco saludables como las gaseosas, energizantes o jugos que contienen sustancias y sabores artificiales o idénticos a los naturales, razones por las cuales se ha ido perdiendo la costumbre de consumirla y por ende, elaborarla. De modo que, solo se prepara en ocasiones especiales o actividades andinas. Es decir, no es fácil conseguirla en lugares de expendio en las ciudades o en tiendas locales. (León, 2008; Maldonado, 2004).
Existen diferentes tipos de chichas, pero todas se elaboran de manera artesanal y tienen procesos muy similares. La fabricación de la chicha de jora se inicia con el tratamiento del maíz con agua mediante remojo por varios días para que pueda germinar. Posteriormente, se procede a secar el maíz germinado, de manera que se pueda obtener harina para ser mezclada con agua, y llevarla a cocción hasta reducirla a un $50 \%$ del volumen inicial. Una vez enfriada, se agrega chancaca o azúcar rubia para el proceso de la fermentación alcohólica con levaduras del género Saccharomyces. Terminada la fermentación, la bebida se filtra, reposa y está lista para consumirse. (Aguirre, 2009).

Dado que en la chicha de jora se utiliza el maíz germinado como materia prima, todos los nutrientes que este posee se transfieren a la bebida para posteriormente ser asimilados por nuestro organismo. El maíz es un cereal y como tal es fuente de fibra y almidones, pero también de proteínas, lípidos y otros hidratos de carbono diferentes al almidón. Asimismo, es fuente de vitaminas del grupo B, y minerales importantes como el calcio y fósforo. Es rica en vitaminas del grupo B, como la vitamina 
B1 necesaria para el desempeńo adecuado del cerebro mediante la absorción de glucosa (proveniente de la dieta) por las neuronas y que se convierte en energía para realizar las actividades diarias y mantener la mente atenta y ágil (Jiménez, 2008). Esta característica se puede observar en el cuadro nutricional que se presenta a continuación.

Tabla 1

Composición nutricional por cada $100 \mathrm{~g}$ de alimento

\begin{tabular}{cccccccccc}
\hline & Kcal & $\begin{array}{c}\text { Chod } \\
\mathrm{g}\end{array}$ & $\begin{array}{c}\text { Proteínas } \\
\mathrm{g}\end{array}$ & $\begin{array}{c}\text { Fibra } \\
\text { cruda } \mathrm{g}\end{array}$ & $\begin{array}{c}\text { Calcio } \\
\mathrm{mg}\end{array}$ & $\begin{array}{c}\text { Fósforo } \\
\mathrm{mg}\end{array}$ & $\begin{array}{c}\text { Tioamina } \\
\mathrm{mg}\end{array}$ & $\begin{array}{c}\text { Riboflavina } \\
\mathrm{mg}\end{array}$ & $\begin{array}{c}\text { Niacina } \\
\mathrm{mg}\end{array}$ \\
\hline $\begin{array}{c}\text { Maíz } \\
\text { alazán } \\
\text { (jora } \\
\text { fresca) }\end{array}$ & 289 & 62,3 & 5,4 & 0,5 & 23 & 275 & 0,28 & 0,4 & 3,4 \\
\hline $\begin{array}{c}\text { Maíz } \\
\text { alazán } \\
\text { (jora } \\
\text { seca) }\end{array}$ & 347 & 73,8 & 7,0 & 1,7 & 38 & 346 & 0,38 & 0,29 & 3,6 \\
\hline $\begin{array}{c}\text { Chicha } \\
\text { de jora }\end{array}$ & 28 & 5,8 & 0,4 & 0,2 & 22 & 18 & 0,02 & 0.1 & 0,2 \\
\hline
\end{tabular}

Nota: INS-Perú. (2009). Tablas peruanas de composición de alimentos

La chicha de jora es un alimento rico en fibra que tiene importancia en la dieta y en nuestro medio social, debido a que produce sensación de llenura, por lo tanto, contribuye a la pérdida de peso y como consecuencia se reducen los niveles de colesterol. Además, colabora con el crecimiento de la flora estomacal, y ayuda al proceso de digestión combatiendo el estreñimiento. También debemos resaltar su aporte de niacina debido a que se trata de un antidepresivo, antidiabético que propicia a la reducción del colesterol (FAO, 2003). La flora intestinal es necesaria para mantener la armonía estomacal, en el cuerpo, a nivel digestivo. Por tanto, se destaca este beneficio de la chicha de jora puesto que es una bebida que posee microflora intestinal conocida como probióticos, los que son capaces de controlar la diarrea y cualquier afección intestinal que implica protección de cualquier bacteria nociva que ingrese a nuestro organismo (Jiménez, 2008).

Como la chicha de jora es una bebida tradicional peruana, su elaboración, predominantemente, de tipo artesanal y dadas sus cualidades nutricionales, amerita realizar trabajos de investigación y desarrollo abocados a estandarizar su proceso de producción, generar productos de bebidas nutritivas y energizantes y su introducción en el mercado juvenil como una alternativa natural y saludable frente al uso de bebidas gaseosas, energizantes y artificiales a base de colorantes y saborizantes. En la presente investigación, se evaluaron diferentes procesos de elaboración de chicha de jora para optimizar los principales parámetros físicos, químicos y microbiológicos que permitan obtener un producto que cumpla con los estándares de calidad.

\section{Materiales y métodos}

\section{Elaboración de chicha de jora}

Se utilizó como materia prima el maíz jora comercial (MJC) y dos tipos de maíz híbrido jaspeado rojo (MHR) y negro (MHN), germinados bajo condiciones de laboratorio. 
La elaboración de la chicha de jora se realizó siguiendo el diagrama de la Figura 1. La materia prima fue seleccionada por inspección para presencia de olores desagradables, mohos, elevada humedad y estado físico de los granos, retirando aquellos que presentaron daño por microorganismos $y / 0$ insectos, determinándosele \% de humedad (NTP 205.037.1975 revisado el 2011) y \% de pérdida por daño. Para la germinación, los granos de maíz MHR y MHN se colocaron en fuentes de acero cubiertas con gasa esterilizada $y$ humedecida en condiciones de semioscuridad a $28^{\circ} \mathrm{C}$ hasta que las raicillas alcanzaron entre 2 a $3 \mathrm{~cm}$ de longitud. El secado se realizó en un deshidratador de cabina horizontal y a una temperatura de $45^{\circ} \mathrm{C}$ por 24 horas y se dio por terminado el proceso con incontenido de humedad final de 7\%.HR y la molienda, en molino de martillo ajustado para obtener gránulos de diámetro de $300 \mu \mathrm{m}$.

Para la obtención del mosto de maíz, se utilizó la metodología descrita por Pomasqui (2012) y por cada litro de preparación se llevó a cocción $200 \mathrm{~g}$ de maíz jora molido por aproximadamente dos (02) horas. Luego se agregaron $50 \mathrm{~g}$ de chancaca y $50 \mathrm{~g}$ de azúcar morena hasta una reducción del volumen de aproximadamente el 50\%. Después el mosto enfriado a $28^{\circ} \mathrm{C}$ fue colado y se guardó en fermentadores de vidrio y añadió $0,5 \mathrm{~g}$ de levadura $S$. cereviciae, previamente activada. Se dejó fermentar de dos a cuatro días hasta la obtención de un $\mathrm{pH}$ de 4,5 y/o un grado alcohólico de 5\% v/v. Durante la fermentación se evaluaron los parámetros de temperatura de fermentación, densidad (método NTP 211.048.2007), pH (método NTP 203.010.2003), grados Brix (método AOAC 931.12.2005) y grado alcohólico (método NTP 210.003.2003). Finalmente, se filtró la chicha de jora y se envasó en botellas de vidrio esterilizadas de $300 \mathrm{~mL}$ de capacidad, que se sometieron a pasteurización a $80^{\circ} \mathrm{C}$ por $5 \mathrm{~min}$, selladas al vacío las que se conservaron a temperatura ambiente hasta su evaluación sensorial.

\section{Determinación de parámetros fisicoquímicos}

Para generar un diagrama de flujo que permita obtener chicha de jora que cumpla con los estándares de calidad, tanto de producto terminado como de procesamiento se evaluaron cuatro parámetros fisicoquímicos: $\mathrm{pH}$, concentración de azúcares reductores ( ${ }^{\circ}$ Brix iniciales), condiciones de germinación y tipo de maíz. En cada una de las pruebas, se evaluaron las variaciones de densidad, grado alcohólico $\left({ }^{\circ} \mathrm{OH}\right), \mathrm{pH}$ y/o ${ }^{\circ}$ Brix.

\section{- Efecto del pH}

Se empleó maíz de jora amarillo, que se comercializa en los mercados de Lima-Perú para preparar mostos de tres valores de $\mathrm{pH}$ inicial: 4,5; 5,0 y 5,5 ajustados con soluciones $1 \mathrm{~N}$ de ácido cítrico y bicarbonato de sodio.

- Efecto de las condiciones de germinación

Se trabajó con dos tipos de condiciones de germinación: artesanal (maíz jora amarillo obtenido de los mercados de la ciudad) y controlado (bajo condiciones de laboratorio a $28^{\circ} \mathrm{C}$ y $\left.95 \% \mathrm{HR}\right)$.

- Efecto de la concentración de azúcares reductores ( ${ }^{\circ} \mathrm{Brix}$ iniciales)

Se probaron mostos con dos concentraciones iniciales de azúcares reductores de 8 y $15,63^{\circ}$ Brix , obtenidos luego de la adición de 50 y $100 \mathrm{~g} / \mathrm{L}$ de azúcar morena, respectivamente. En 
ambos casos, se mantuvo la cantidad estándar de $50 \mathrm{~g}$ de chancaca que también aporta azúcares reductores.

\section{- Efecto del tipo de maíz}

Para evaluar el efecto del tipo de maíz, se utilizaron tres tipos de maíz germinado: jora de maíz amarillo comercial y jora de maíz rojo y negro germinados en condiciones de laboratorio

\section{Análisis estadístico}

Para calcular el efecto del pH, las condiciones de germinación, la concentración de azúcares reductores y el tipo de maíz jora sobre los otros parámetros se utilizó el análisis de varianza (ANOVA) y la prueba de Tukey con un nivel de significancia del 5\%, a través del software MINITAB 17.

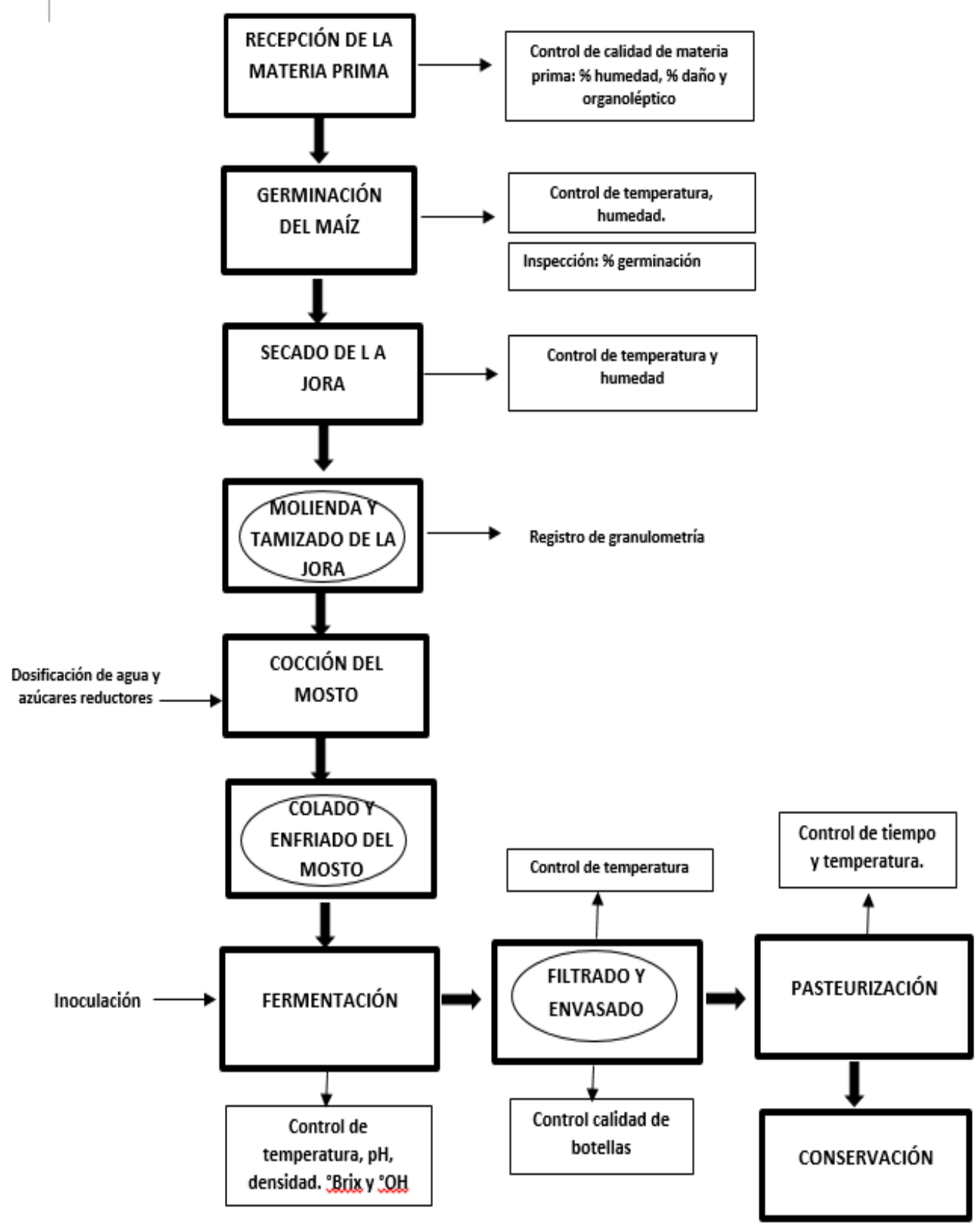

Figura 1. Diagrama de flujo de la elaboración de chicha de jora y parámetros o métodos de control que se ejecutaron. 


\section{Caracterización de la chicha de jora}

Cada uno de los diferentes productos de chicha de jora obtenidos, fueron sometidos a análisis de calidad microbiológica y sensorial para verificar que se cumplieran con los parámetros establecidos para este producto. Se tuvo en cuenta, además, que los requisitos de la chicha de jora son: color pardo claro, aroma agradable y sabor agridulce que deben mantenerse en el tiempo, textura ligeramente viscosa y con trazas de sólidos suspendidos, grado de claridad turbio y niveles de carga microbiana aceptables (Tabla 2).

Para obtener la calidad microbiológica, se emplearon los métodos estandarizados ISO 4833 (Recuento de Aerobios Mesófilos Viables) e ISO 7954 (Recuento de Mohos y Levaduras). Sensorialmente, cuatro muestras de chicha de jora obtenidas bajo iguales condiciones de laboratorio en los parámetros de temperatura, $\mathrm{pH}$, tiempo, ${ }^{\circ}$ Brix y densidad y una muestra adquirida comercialmente como control (chicha de jora del restaurante Incahuasi) fueron codificadas: Amarillo con raicillas (7621), Amarillo sin raicillas (1432), Rojo (0860), Negro (9421) e Incahuasi (5734); y presentadas a un panel de trece jueces semientrenados para una evaluación de intensidad de sabor y de preferencia, emplear una prueba escalar de control de cinco puntos de intensidad creciente. Finalmente, la muestra de chicha de jora de mayor preferencia fue luego evaluada por un panel de siete jueces entrenados para los perfiles de sabor, olor y textura/consistencia del producto final. Los datos sensoriales fueron evaluados, de manera gráfica y estadística mediante análisis de varianza.

Tabla 2

Niveles microbiológicos máximos permitidos, según MR 591-2008 MINSA

\begin{tabular}{lc}
\hline Agente microbiano & Límite máximo (UFC/mL) \\
\hline Aerobios mesófilos viables & 10 \\
\hline Mohos & 5 \\
\hline Levaduras & 10 \\
\hline
\end{tabular}

\section{Resultados}

\section{Inspección de materia prima}

Del maíz jora adquirido comercialmente, se rechazó el 8,5\% por daño biológico (insectos) y el 1,5\% del obtenido bajo condiciones de laboratorio por ausencia de germinación. El contenido de humedad fue de 13,8\% para maíz jora obtenido comercialmente y $10,6 \%$ para maíz jora generado en laboratorio tanto rojo como negro.

\section{Efecto del pH}

Durante el seguimiento de los procesos de fermentación, la temperatura de la misma fue de $28,5 \pm 0,5^{\circ} \mathrm{C}$. Los ${ }^{\circ}$ Brix se redujeron en un $37 \%$ para los pH 4,5 y 5,5 y un $48 \%$ para el pH 5,0. En los tres casos, la densidad se redujo en un $91 \%$ y el descenso del $\mathrm{pH}$ promedio fue de 1,7 obteniéndose chichas de jora con $\mathrm{pH} 4,13 \pm 0,3$ luego de $48 \mathrm{~h}$ de fermentación. La densidad y los grados Brix presentaron igual grado de fluctuación a lo largo del tiempo de fermentación. En el caso de los grados alcohólicos, la fluctuación 
fue menos marcada a $\mathrm{pH} 5,0$ y 5,5 , pero la producción de etanol $\left({ }^{\circ} \mathrm{OH}\right)$ fue mayor a pH 5,5 (Figura 2). Estadísticamente, para los tres valores de $\mathrm{pH}$ inicial, las diferencias, antes mencionadas no fueron significativas (Tabla 3).

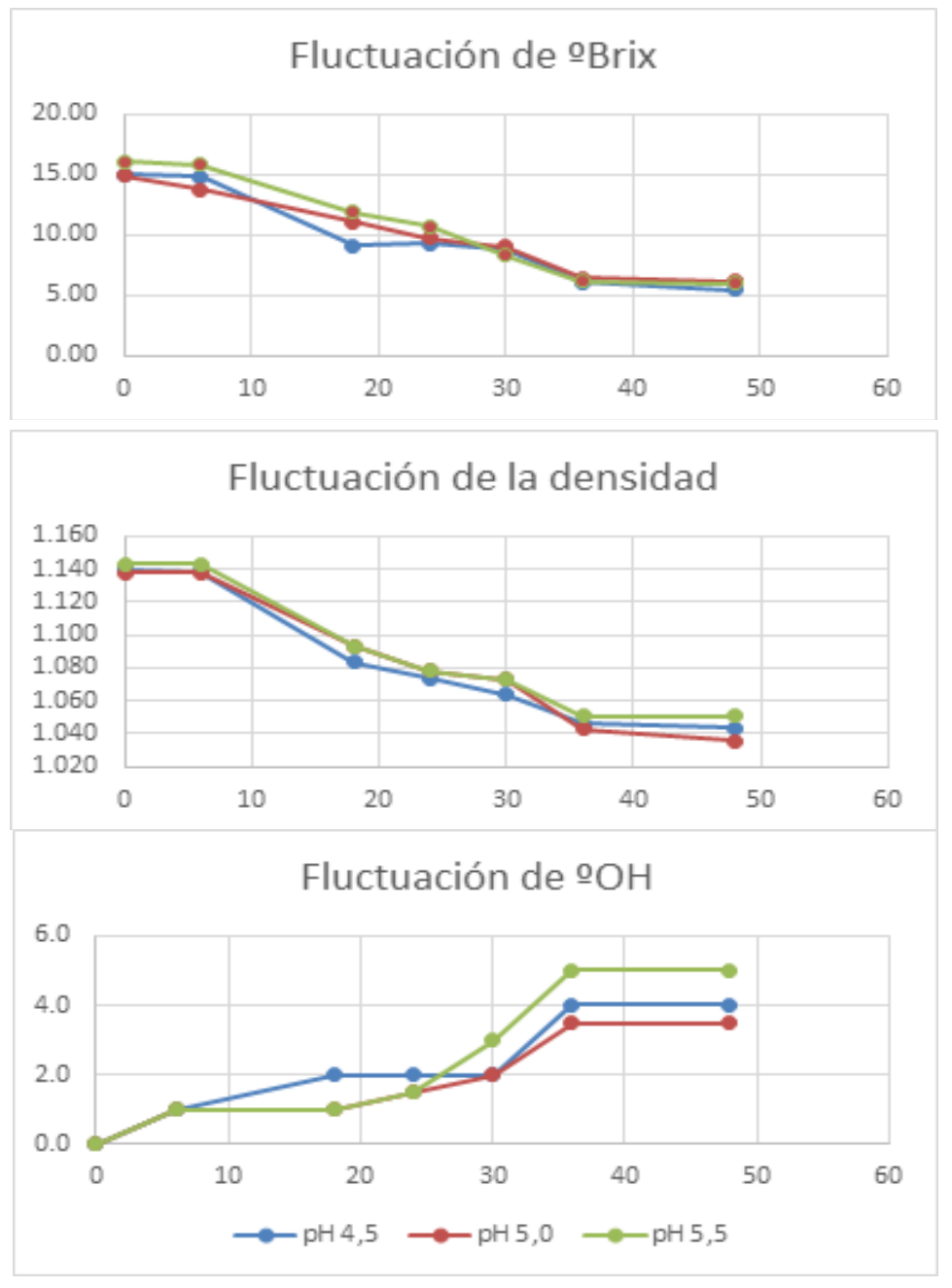

Figura 2. Efecto del $\mathrm{pH}$ inicial sobre los ${ }^{\circ}$ Brix, los grados alcohólicos $\left({ }^{\circ} \mathrm{OH}\right)$ y la densidad del proceso de fermentación de maíz jora.

Tabla 3

Análisis estadístico de tres parámetros químicos del proceso de fermentación en tres valores iniciales de $\mathrm{pH}$ del mosto de maiz jora.

\begin{tabular}{|c|c|c|c|c|c|c|c|}
\hline \multirow{3}{*}{ Tratamiento } & \multirow{3}{*}{$\mathbf{N}$} & \multicolumn{6}{|c|}{ Factores } \\
\hline & & \multicolumn{2}{|c|}{ Densidad } & \multicolumn{2}{|c|}{${ }^{\circ}$ Brix } & \multicolumn{2}{|c|}{${ }^{\circ} \mathrm{OH}$} \\
\hline & & $\overline{\mathrm{X}}$ & St.Dev & $\overline{\mathrm{X}}$ & St.Dev & $\overline{\mathrm{X}}$ & St.Dev \\
\hline $\mathrm{pH} 4,5$ & 7 & $1,0841 \mathrm{~A}$ & 0,0403 & $9,7860 \mathrm{~A}$ & 3,816 & $2,143 \mathrm{~A}$ & 1,464 \\
\hline $\mathrm{pH} 5,0$ & 7 & $1,0855 \mathrm{~A}$ & 0,0410 & $10,164 \mathrm{~A}$ & 3,372 & $1,786 \mathrm{~A}$ & 1,318 \\
\hline $\mathrm{pH} 5,5$ & 7 & $1,0901 \mathrm{~A}$ & 0,0391 & $10,714 \mathrm{~A}$ & 4,194 & $2,357 \mathrm{~A}$ & 2,015 \\
\hline
\end{tabular}

Nota: Promedios que no comparten una misma letra son, significativamente, diferentes 


\section{Efecto de la concentración inicial de azúcares $\left({ }^{\circ}\right.$ Brix $)$}

Aproximadamente, el 67\% del azúcar presente en el mosto se convirtió en alcohol etílico y anhídrido carbónico. La conversión fue drástica durante las primeras 42 horas y se mantuvo hasta por 66 horas (dos días 18 horas). Este consumo de azúcar se correlacionó con un descenso del $\mathrm{pH}$ y de la densidad en un 80 y $86 \%$, respectivamente. Se obtuvieron 5 y $6^{\circ}$ alcohólicos según se trabajó con 8,0 o 15,63 ${ }^{\circ}$ Brix iniciales, respectivamente (Figura 3). Estadísticamente, la variación de ${ }^{\circ}$ Brix iniciales, en los parámetros evaluados, no fue significativa para $\mathrm{pH}$ y el grado alcohólico, sí lo fue para la densidad (Tabla 4).

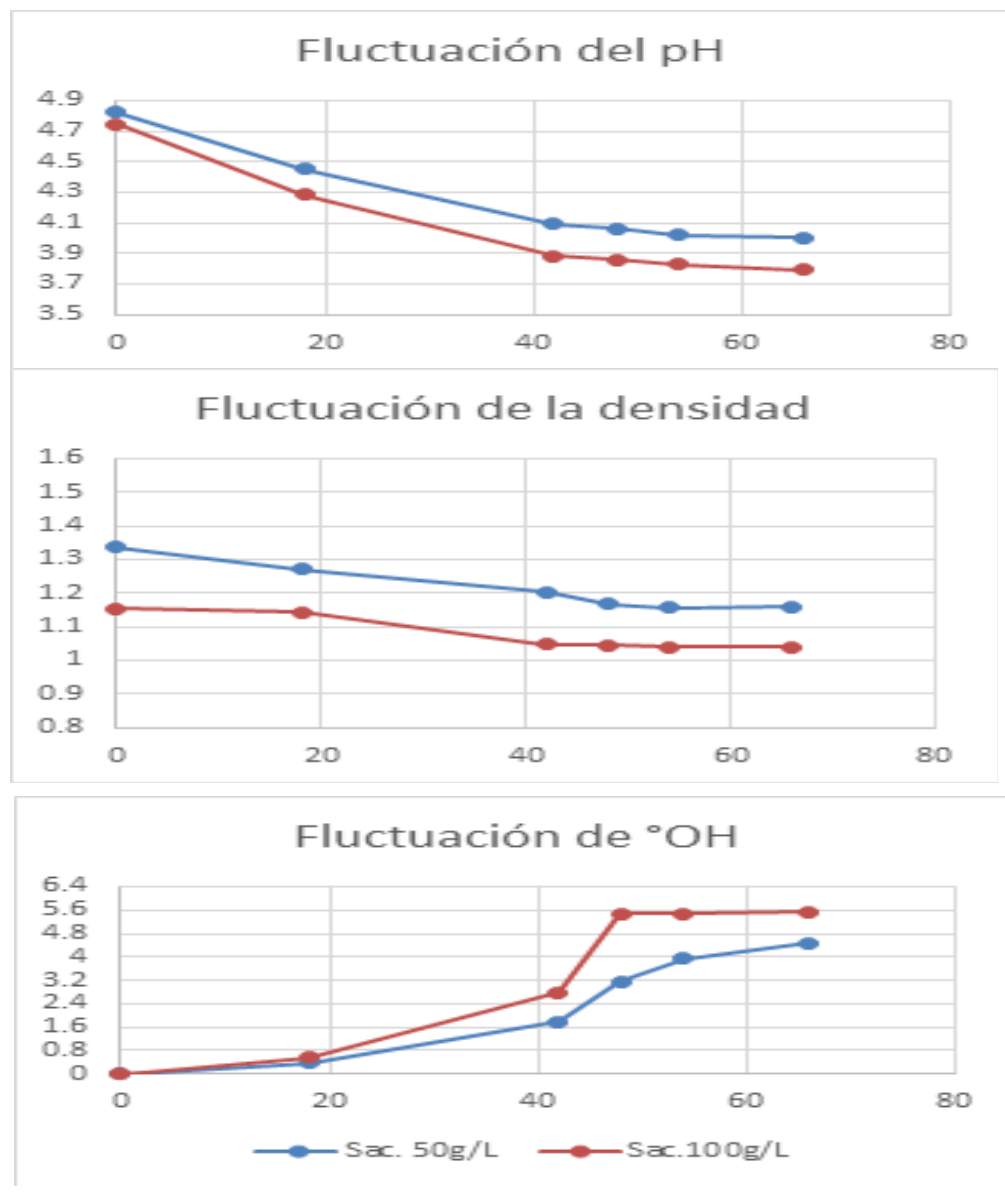

Figura 3. Efecto de la concentración de azúcares ( ${ }^{\circ}$ Brix iniciales) sobre el $\mathrm{pH}$, la densidad y los grados alcohólicos $\left({ }^{\circ} \mathrm{OH}\right)$ del proceso de fermentación de maíz jora

Tabla 4

Análisis estadistico de tres parámetros quimicos del proceso de fermentación en dos valores iniciales de azúcar moreno $(g / L)$

\begin{tabular}{|c|c|c|c|c|c|c|c|}
\hline \multirow{3}{*}{ Tratamiento } & \multirow{3}{*}{$\mathbf{N}$} & \multicolumn{6}{|c|}{ Factores } \\
\hline & & \multicolumn{2}{|c|}{$\mathrm{pH}$} & \multicolumn{2}{|c|}{ Densidad } & \multicolumn{2}{|c|}{${ }^{\circ} \mathrm{OH}$} \\
\hline & & $\overline{\mathrm{X}}$ & St.Dev & $\overline{\mathrm{X}}$ & St.Dev & $\overline{\mathrm{X}}$ & St.Dev \\
\hline Sac. $50 \mathrm{~g} / \mathrm{L}$ & 6 & $4,242 \mathrm{~A}$ & 0,331 & $1,2254 \mathrm{~A}$ & 0,0752 & $2,290 \mathrm{~A}$ & 1,867 \\
\hline Sac. $100 \mathrm{~g} / \mathrm{L}$ & 6 & $4,065 \mathrm{~A}$ & 0,379 & $1,0780 \mathrm{~B}$ & 0,0549 & $3,30 \mathrm{~A}$ & 2,57 \\
\hline
\end{tabular}

Nota: Promedios que no comparten una misma letra son, significativamente, diferentes 


\section{Efecto del tipo de maíz}

Los valores iniciales de $\mathrm{pH},{ }^{\circ}$ Brix y densidad del mosto fueron inferiores para el maíz morocho. El comporta- miento cinético del mosto de maíz negro y del maíz rojo fue similar entre sí, pero diferente al del maíz morocho (Figura 4). Estadísticamente, la diferencia de sus medias no fue significativa (Tabla 5).
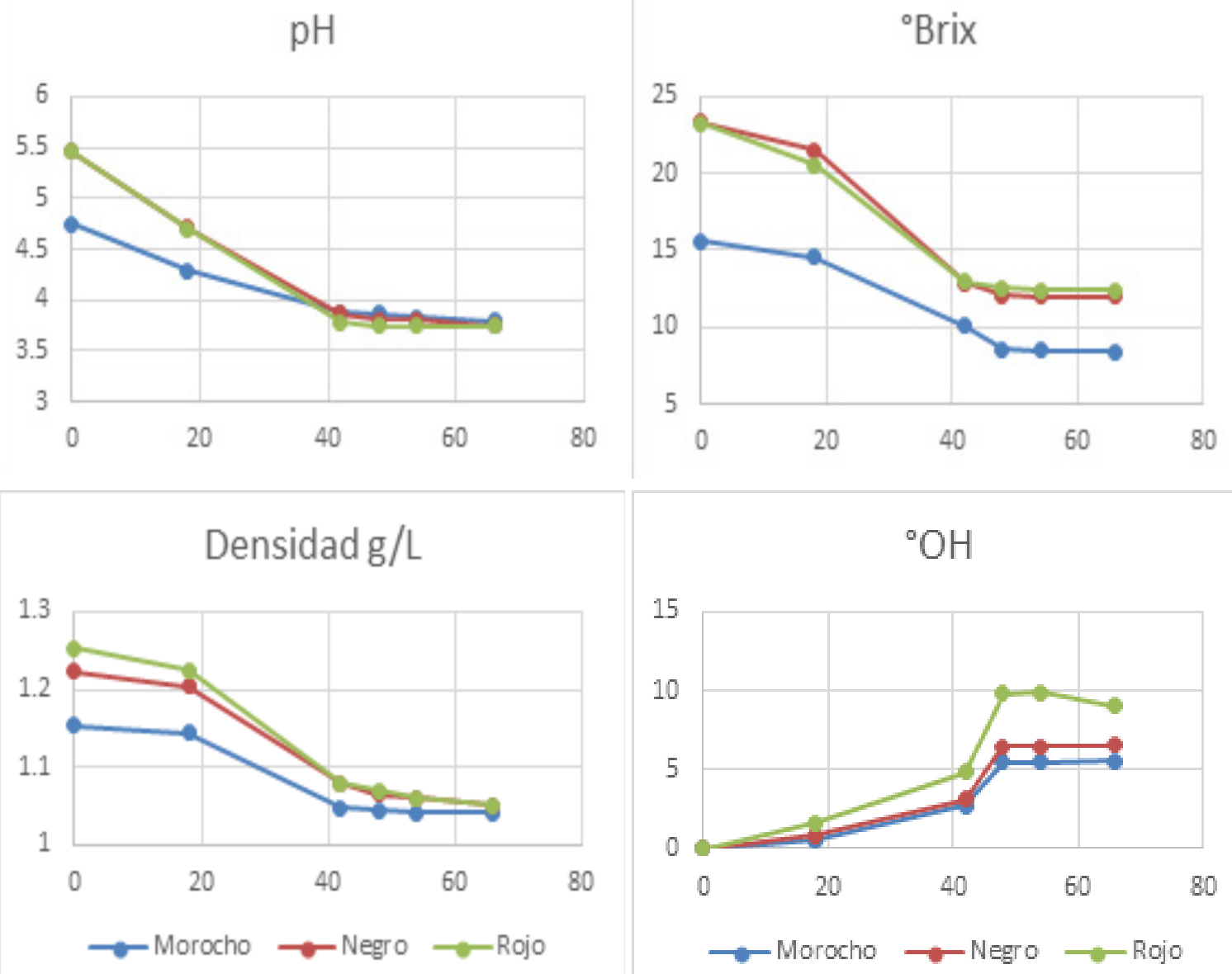

Figura 4. Efecto del tipo de maíz germinado sobre el $\mathrm{pH}$, ${ }^{\circ}$ Brix, densidad y ${ }^{\circ} \mathrm{OH}(\mathrm{C})$ del proceso de fermentación de maíz jora

Tabla 5

Análisis estadístico de tres parámetros químicos del proceso de fermentación de tres tipos de maiz jora

\begin{tabular}{|c|c|c|c|c|c|c|c|}
\hline \multirow{3}{*}{ Tratamiento } & \multirow{3}{*}{$\mathbf{N}$} & \multicolumn{6}{|c|}{ Factores } \\
\hline & & \multicolumn{2}{|c|}{ pH } & \multicolumn{2}{|c|}{${ }^{\circ} \mathrm{Brix}$} & \multicolumn{2}{|c|}{${ }^{\circ} \mathrm{OH}$} \\
\hline & & & St.Dev & & St.Dev & & St.Dev \\
\hline Maíz morocho & 9 & $4,1963 \mathrm{~A}$ & 0,4132 & $12,204 \mathrm{~A}$ & 3,421 & $2,202 \mathrm{~A}$ & 2,487 \\
\hline Maíz negro & 9 & $4,4625 \mathrm{~A}$ & 0,7836 & $17,465 \mathrm{~A}$ & 5,801 & $2,615 \mathrm{~A}$ & 2,825 \\
\hline Maíz rojo & 9 & $4,4213 \mathrm{~A}$ & 0,8198 & $17,333 \mathrm{~A}$ & 5,397 & $4,095 \mathrm{~A}$ & 4,345 \\
\hline
\end{tabular}

Nota: Promedios que no comparten una misma letra son, significativamente, diferentes 


\section{Caracterización microbiológica y sensorial}

Microbiológicamente, la chicha de jora producto de los diferentes procesos de fermentación cumplió con los parámetros establecidos por DIGESA para una bebida fermentable de consumo humano. Sensorialmente, las cuatro muestras de chicha de jora obtuvieron entre 22 y 53 puntos (Tabla 6). Estos resultados fueron evaluados, estadísticamente, mediante prueba de Análisis de Varianza de Friedman (Tabla 7), determinándose que el grado de preferencia para las muestras fue, estadísticamente, diferente con un valor $\delta$ de 16 ; siendo la muestra del código 0860 (jora de maíz negro) la de mayor preferencia y la muestra del código 5734 (chicha de Incautase) la de menor preferencia (Tabla 8).

Tabla 6

Evaluación sensorial de cinco muestras de chicha de jora mediante prueba escalar de control de cinco puntos

\begin{tabular}{|c|c|c|c|c|c|c|}
\hline \multirow{2}{*}{ Juez } & \multicolumn{5}{|c|}{ Código/Valoración } & \multirow{2}{*}{ Total } \\
\hline & 0860 & 1432 & 7621 & 9421 & 5734 & \\
\hline 1 & 4 & 1 & 3 & 5 & 2 & 15 \\
\hline 2 & 3 & 4 & 2 & 5 & 1 & 15 \\
\hline 3 & 5 & 1 & 4 & 3 & 2 & 15 \\
\hline 4 & 4 & 2 & 5 & 3 & 1 & 15 \\
\hline 5 & 3 & 1 & 4 & 5 & 2 & 15 \\
\hline 6 & 2 & 5 & 3 & 4 & 1 & 15 \\
\hline 7 & 5 & 2 & 1 & 4 & 3 & 15 \\
\hline 8 & 4 & 3 & 2 & 5 & 1 & 15 \\
\hline 9 & 5 & 4 & 2 & 3 & 1 & 15 \\
\hline 10 & 5 & 2 & 4 & 3 & 1 & 15 \\
\hline 11 & 3 & 1 & 2 & 5 & 4 & 15 \\
\hline 12 & 5 & 3 & 4 & 2 & 1 & 15 \\
\hline 13 & 5 & 1 & 4 & 3 & 2 & 15 \\
\hline Total & 53 & 30 & 40 & 50 & 22 & \\
\hline
\end{tabular}

Tabla 7

Análisis de Varianza de Friedman de los valores de preferencia de cinco muestras de chicha de Jora con un $\alpha$ de 0,05

\begin{tabular}{|l|l|l|l|l|}
\hline $\mathbf{N}^{\circ}$ de juicios & 13 & Chi $_{\text {calculada }}$ & Chi $_{\text {tabulada }}$ & Valor $\boldsymbol{\delta}$ \\
\hline $\mathbf{N}^{\circ}$ de muestras & 5 & 21,2 & 9,49 & 16 \\
\hline G. 1. & 4 & & & \\
\hline
\end{tabular}


Asimismo, el panel de jueces entrenados estableció el perfil de textura, aroma y sabor de la muestra de mayor preferencia (código 0860), generándose la gráfica mostrada en la Figura 5, donde se puede establecer que es una chicha agradable de sabor dulce, pero ligeramente ácida, de mediano grado alcohólico, consistencia fluida con muy poca astringencia y con aroma a malta fermentada, ligeramente afrutada.

Tabla 8

Comparación de la diferencia estadistica entre la preferencia de las cinco muestras de chicha de jora evaluadas, sensorialmente

\begin{tabular}{cccccccc}
\hline \multirow{2}{*}{ Código } & Código & 0860 & 1432 & 7621 & 9421 & 5734 & \multirow{2}{*}{ TOTAL } \\
& Suma categ. & 53 & 30 & 40 & 50 & 22 & \\
\hline 860 & 53 & 0 & 23 & 13 & 3 & 31 & 70 \\
\hline 1432 & 30 & -23 & 0 & -10 & -20 & 8 & -45 \\
\hline 7621 & 40 & -13 & 10 & 0 & -10 & 18 & 5 \\
\hline 9421 & 50 & -3 & 20 & 10 & 0 & 28 & 35 \\
\hline 5734 & 22 & -31 & -8 & -18 & -28 & 0 & -86 \\
\hline
\end{tabular}

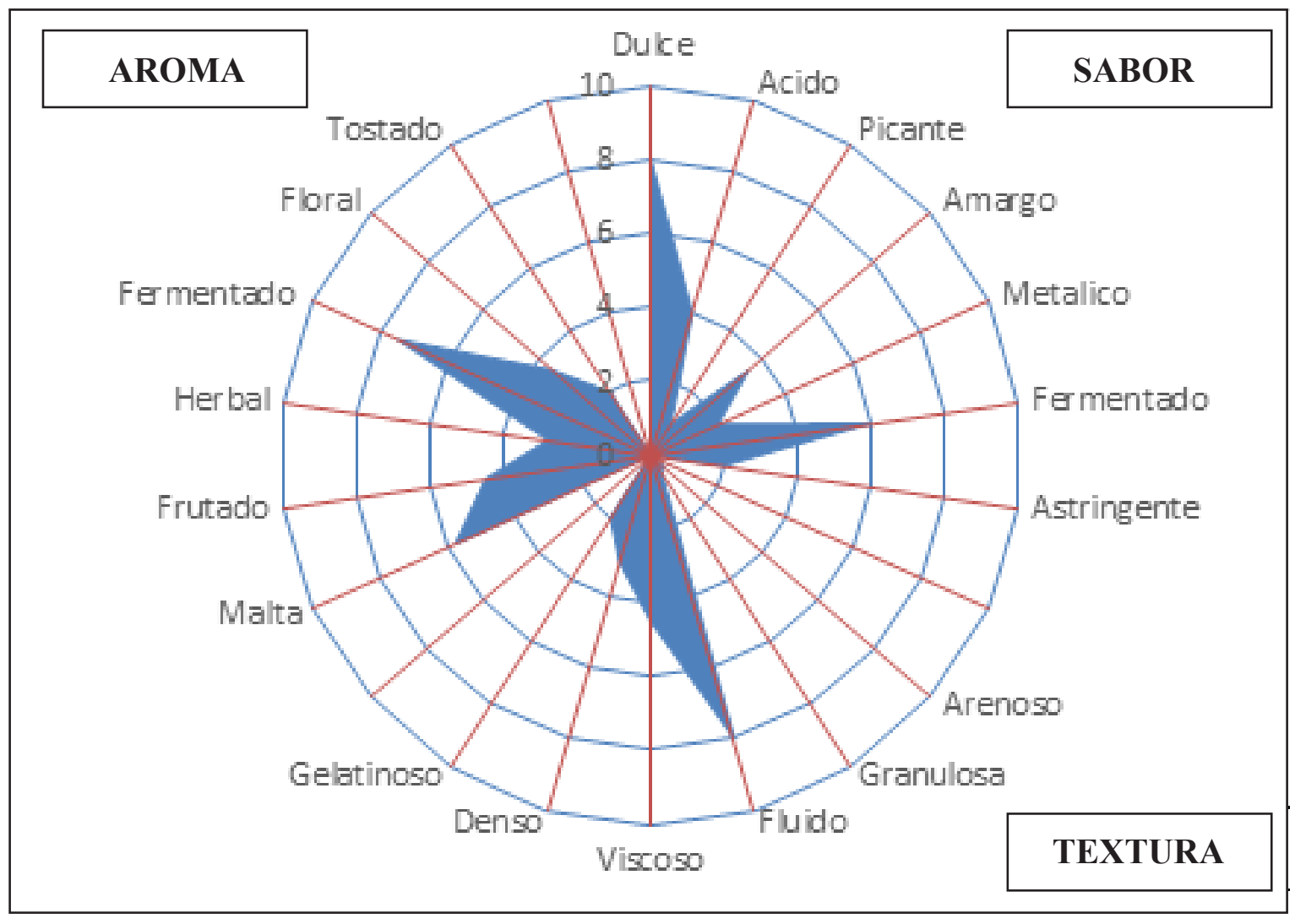

Figura 5. Perfil de aroma, sabor y textura de la chicha de jora de maíz negro sin tostar (código 0860) 


\section{Discusión}

\section{Efecto del pH}

Los valores de $\mathrm{pH}$ de un proceso de fermentación son importantes por su efecto sobre la levadura, y el color, sabor y turbidez de la chicha de jora (Rojas, 2013). El nivel de acidez o basicidad que contenga el medio en el que se encuentran las levaduras que promuevan la producción de alcohol, determinará el nivel de consumo de sustrato, así como la velocidad de fermentación. La actividad de las levaduras disminuye cuando se encuentra a niveles de $\mathrm{pH}$ bajos. Por lo que en un $\mathrm{pH}$ óptimo es donde el metabolismo de las levaduras es satisfactorio para promover un buen consumo del sustrato.

Según varias investigaciones, el pH óptimo para el crecimiento de las levaduras, en la fermentación alcohólica, oscila en el rango de 3 y 5 (Granadillo \& Col., 2014; Ward, 1991). Además, en este intervalo de $\mathrm{pH}$, se elimina la competencia por el azúcar y los nutrientes por parte de las bacterias presentes en el mosto. Asimismo, López (2010) citado por Rojas (2013) indica que la fermentación de la jora debe darse hasta obtener un $\mathrm{pH} 4$, acidez $0,4 \%$ (ácido láctico) y un grado alcohólico de 2 para asegurar que este producto tenga un largo periodo de vida. Para los mostos con los tres valores iniciales de $\mathrm{pH}$ probados, luego de dos días de fermentación, se alcanzaron valores finales de $\mathrm{pH}$ de 4,13 \pm 0,3 que permitieron una intensa fermentación y una mayor producción de etanol $\left(4,5^{\circ} \mathrm{OH}\right.$ en promedio) que el indicado por López. La fermentación del mosto con $\mathrm{pH}$ inicial de 5,5 fue el que generó una mayor cantidad el etanol en el tiempo de prueba, que permitió obtener los $4^{\circ}$ especificados en tan solo 36 horas de fermentación. Por estas razones, se asume que 5.5 como $\mathrm{pH}$ óptimo del mosto para producción de chicha de jora.

\section{Efecto de la concentración de azúcares reductores ( $\mathrm{B}$ Brix iniciales)}

Los sistemas de fermentación de las levaduras requieren como principales nutrientes una fuente de carbono o glucosa. Sin embargo, una alta concentración de este último sustrato puede inhibir el crecimiento de la levadura y la fermentación como resultado de una elevada presión osmótica. Este efecto, puede ocurrir en concentraciones de azúcares fermentables en el rango de 125 y $250 \mathrm{~g} / \mathrm{l}$ (López et al. 2009). Las cantidades de azúcares probados se encuentran por debajo de los rangos brindados como límites por López et al. \& Col. (2009), por lo que se presentó una fermentación tumultuosa del mosto tornándose turbio y espumoso por el $\mathrm{CO}_{2}$ que se desprendía, similar a lo presentado por otras investigaciones (León \& Zapata, 2008; Rojas, 2013).

El resultado final después del proceso fermentativo es la presencia de etanol en la bebida de jora, el cual se produce por la formación del piruvato por glicólisis en aerobiosis y su posterior descarboxilación, producida en ausencia del oxígeno para terminar con dos moléculas de aldehídos y finalmente dos moles de etanol (León, 2008; Harmon, 2012). Los azúcares utilizados en la producción de piruvato provienen del almidón contenido en los granos de maíz y que es catabolizado por enzimas hidrolíticas del grano, durante las etapas de remojo y germinación, hasta tener únicamente el azúcar que será el sustrato para la fermentación (Sánchez \& Col., 2010; Teijón et.al., 2006). 
La velocidad del proceso fermentativo mostró una disminución conforme la producción de etanol superaba los $4^{\circ} \mathrm{OH}$. (Figuras 3 y 4 ). Esto se debe a que la membrana citoplasmática cumple una función importante ayudando a la célula, en su lucha contra los efectos del etanol y de la acidez del medio que la rodea. Esta membrana por sí sola no es permeable a macromoléculas o iones con excepción de los protones $\left(\mathrm{H}^{+}\right)$. La acumulación de etanol en el medio de fermentación interacciona con el funcionamiento de la membrana plasmática. El principal efecto del etanol es hacer que la membrana permita el paso de protones e inhibir la actividad de la bomba de protones ATPasa, la cual se encarga de mantener la homeóstasis del pH de la levadura. Por lo tanto, las células se encuentran en la obligación de consumir más energía para mantener la homeostasis de $\mathrm{pH}$, hasta que el equilibrio entre la entrada pasiva de electrones y su expulsión por la actividad de la bomba de protones ATPasa no pueda ser mantenido dando lugar a la muerte de la célula (Salmón et al., 2007).

Teniendo en cuenta la importancia de la concentración de etanol, en la producción de chicha de jora, y que esta no es afectada significativamente por la cantidad de sacarosas probada (Tabla 4), se establece como concentración inicial óptima de azúcares reductores, los aportados por chancaca y azúcar morena $50 \mathrm{~g} / \mathrm{L}$ cada una.

\section{Efecto del tipo de maíz}

El comportamiento cinético similar entre el mosto del maíz negro y rojo se debería a que los germinados se originaron en el laboratorio; en cambio, el de maíz morocho fue adquirido en el co- mercio y por lo tanto, es difícil precisar el tiempo transcurrido desde su producción hasta su adquisición y uso en este trabajo.

La producción de etanol fue mayor para ambos tipos de jora germinada en el laboratorio, lo cual se relaciona con su mayor contenido de oBrix. que representa la cantidad de sólidos totales, entre ellos, los azúcares fermentables a partir de los cuales $S$. cereviciae produce etanol, entonces, el proceso de germinación bajo condiciones controladas permitió una mayor conservación de azúcares fermentables lo que mejoró la velocidad de dichos azúcares que pasaron a etanol por el microorganismo. Los azúcares fermentables que se pueden hallar en la chicha de jora suelen ser: dextrosa, glucosa, azúcar invertido y menos frecuentemente maltosa y lactosa; estos son fácilmente metabolizados por las levaduras, y la maltosa es la más catabolizada (García \& Mamani, 2008; Pomatsqui, 2012; ).

Si bien, la jora de maíz negro y la de maíz rojo se iniciaron con igual cantidad de azúcares (aprox. $23^{\circ}$ Brix), la composición de estos azúcares fue diferente. Los azúcares presentes en un mosto de jora son de dos tipos: fermentables y no fermentables y sus proporciones varían no solo de acuerdo con el tipo de maíz, sino que también cambian de cosecha a cosecha de un mismo tipo de maíz, al ser influenciado por las condiciones de cultivo. Como ambos tipos de jora se generaron en el laboratorio al mismo tiempo y bajo las mismas condiciones ambientales, los efectos de las condiciones de procesado quedan minimizadas, por lo que se puede establecer que la cantidad de azúcares fermentables, presentes en el mosto de maíz rojo, fue mayor en la jora de maíz negro y 
que esto se debería, en gran parte, al tipo de maíz.

\section{Caracterización microbiológica y sen- sorial}

El análisis sensorial de los alimentos es un instrumento eficaz para el control de calidad y aceptabilidad de un alimento, y si el mismo se quiere comercializar, debe cumplir los requisitos mínimos de higiene, inocuidad y calidad del producto para que este sea aceptado por el consumidor, más aún cuando debe ser protegido por un nombre comercial los requisitos son mayores, ya que debe poseer las características que justifiquen su reputación como producto comercial.

En general, el análisis se realiza con el fin de encontrar la fórmula adecuada que agrade al consumidor, buscar también la calidad, e higiene del alimento para que tenga éxito en el mercado (Sulco \& Alvarado, 2011). Típicamente, el color de la chicha es pardo oscuro o un color semejante a este, el aroma es característico de la chicha, pudiendo clasificarla como un aroma propio inconfundible con cualquier otro producto, el sabor es descrito como agridulce con una ligera sensación del gas producido por la fermentación, aunque no tan fuerte, la bebida en general es turbia y puede incluso contener pequeñas cantidades de sedimento. (León \& Zapata, 2008). Todas las chichas de jora, elaboradas en este trabajo de investigación presentaron aspecto turbio y luego de la pasteurización, la formación de sedimento durante su conservación. El grado de claridad de la chicha de jora, se debe a que mientras el maíz se someta a mayor tiempo de cocción se producirá un aumento en la extracción de los com- ponentes solubles de la jora, el aspecto turbio y la presencia de sedimentación, indica que hubo precipitación de los sólidos insolubles: gomas, proteínas, levaduras, cuando la fermentación ha terminado, debido a que las proteínas reaccionan con los polifenoles presentes en la malta y de esta manera, da lugar a compuestos menos solubles, de alto peso molecular, que son una de las causas principales del enturbiamiento de la chicha de jora (Fula, 2010).

Sensorialmente, la chicha de jora codificada como 0860 obtenida a partir de la fermentación de jora de maíz negro, fue la de mayor aceptación por el panel de jueces. Chicha que organolépticamente fue agradable de sabor dulce, pero ligeramente ácido, de mediano grado alcohólico, consistencia fluida con muy poca astringencia y con aroma a malta fermentada ligeramente afrutada. Durante la fermentación de la malta, junto con la producción de dióxido de carbono y etanol, se genera una amplia variedad de compuestos de vital importancia para lo que se conoce como "flavor", es decir, el sabor y el aroma. Entre ellos, los ésteres conforman el grupo de compuestos que inciden de manera significativa en la calidad sensorial del producto final. En cervezas se han detectado más de 100 ésteres diferentes. Los más relevantes, generados por las levaduras a través de su metabolismo secundario, pueden dividirse en dos grupos principales; ésteres de acetato, tales como etil acetato, isoamil acetato y feniletil acetato, y ésteres de etilo, como etil hexanoato y etil octanoato. Ambos grupos aromas y sabores florales frutales y contribuyen a su característica complejidad se aroma y sabor. Algunos ésteres en concentraciones muy altas aportan 
aromas y sabores indeseados (p.ej., acetato de etilo: solvente). Además, la presencia de diferentes ésteres puede tener un efecto sinérgico e influir en la percepción sensorial general, a pesar de que sus concentraciones individuales estén por debajo del umbral (Gamero et al., 2014; Verstrepen et al., 2003).

Los esteres generados por las levaduras se producen por reacción enzimática entre un ácido orgánico y un alcohol y es regulado por factores como las enzimas, las condiciones de fermentación, la composición del mosto y las características de las levaduras (Loviso \& Libkind, 2018). En ausencia de oxígeno, la reacción entre acetil-coA y un alcohol (etanol o alcoholes superiores) permite la formación de ésteres de acetato, mientras que la combinación entre las largas cadenas de acil-coA y etanol produce etil ésteres (Blieck et al., 2007). Las enzimas alcohol acetil transferasas I y II, codificadas por los genes atf1 y atf2, respectivamente, son las responsables de la formación de ésteres de acetato. Los genes atf1 y atf2 han sido identificados en el genoma de Saccharomyces cerevisiae (Saerens et al., 2010). Asimismo, Dekonink et al. (2012) han demostrado que la adición de sacarosa al mosto, aunque posee un efecto estimulante en la expresión del gen atfl, genera menores concentraciones de ésteres de acetato en relación con el agregado de maltosa.

Por lo tanto, el sabor y aroma que caracterizaron a la chicha de jora de mayor aceptabilidad estuvieron generados, primordialmente, por ésteres de acetato derivados del metabolismo de los azúcares presente en su mosto; azúcares aportados tanto por la chancaca como por el azúcar adicionado, pero también por los azúcares contenidos en el maíz. Estos últimos serían los que marcaron la diferencia en aceptabilidad con respecto a las otras chichas evaluadas, ya que todos los mostos fueron procesados de igual modo y a todos se le agregaron los mismos ingredientes a excepción del tipo de maíz.

\section{Conclusiones}

Tras el análisis de los resultados de este estudio se concluye que:

- Los parámetros del proceso de fermentación a $30^{\circ} \mathrm{C}$ que conducen a la obtención de chicha de jora con gran aceptabilidad son: mostos con $15,63^{\circ}$ Brix, pH 5,5 y densidad entre 1,15 y $1,35 \mathrm{~g} / \mathrm{L}$. Los obtenidos de maíz jora germinado bajo condiciones controladas, con la adición de $100 \mathrm{~g} / \mathrm{L}$ de chancaca y $50 \mathrm{~g} / \mathrm{L}$ de azúcar morena y fermentados por $60 \mathrm{a}$ 72 horas para obtener una chicha de pH 4 a 4,3 y 4 a $5^{\circ} \mathrm{OH}$.

- De las pruebas sensoriales, se tiene que la chicha de jora obtenida de jora de maíz negro tiene mayor preferencia respecto al perfil de textura, aroma y sabor que las obtenidas de jora de maíz rojo y morocho, y se obtuvo similar resultado de preferencia en relación con la chicha de jora comercializada por la franquicia INCAHUASI.

- Microbiológicamente, la chicha de jora obtenida en los diferentes procesos de fermentación, cumple con los parámetros establecidos por GIGESA para una bebida fermentable de consumo humano. 


\section{Referencias}

Arroyo-López, F.N, Orlic, S., Querol, A. \& Barrio, E. (2009). Effects of temperature, $\mathrm{pH}$ and sugar concentration on the growth parameters of Saccharomyces cerevisiae, S. kudriavzevii and their interspecific hybrid. International Journal of Food Microbiology, 131(2-3), 120-127.

Biasutti, C. \& Galiñanes, V. (2001). La germinación de semillas de maíz (Zea mays L.) bajo estrés hídrico. Relaciones entre caracteres de plántula con el rendimiento a campo. Revista Agrocientia, 17, 37 $-44$.

Blieck, L., Toye, G., Dumortier, F., Vertrepen, K.J, Delvaux, F.D. \& Thevelein, J.M.V.P. (2007). Isolation and characterization of brewer's yeast variants with improved fermentation performance under highgravity conditions. Applied and Environmental Microbiology, 73, $815-824$.

Dekoninck, T.M., Verbelen, P.J., Delvaux, F., Van Mulders, S.E. \& Delvaux, F.R. (2012). The importance of wort composition for yeast metabolism during accelerated brewery fermentations. Journal of the American Society of Brewing Chemists, 7, $195-204$.

Food and Agriculture Organization of the United Nations. (1995). Norma para el maiz (CODEX STAN 153-1985). Recuperado de la página del FAO: http://www.fao. org/fao-who-codexalimentarius/ codex-texts/list-standards/es/
Fula, A.G. (2014). Desarrollo de una bebida fermentada con adición de cocción de maíz. Especialización en ciencia y tecnología de alimentos (tesis de maestría). Universidad Nacional de Colombia, Colombia. Recuperado de http://www.bdigital.unal.edu. co/2387/1/107391.2010.pdf

Gamero, A., Ferreira, V., Pretorius, I.S. \& Querol, A. (2014). Wine, beer and cider: Unravelling the aroma profile. En J. Piskur, C. Compagno (eds.). Molecular Mechanisms in Yeast Carbon Metabolism, pp. 261-297. Alemania: Springer Heidelberg.

García, D. \& Mamani, G. (2008). Selección de levaduras nativas Saccharomyces cerevisiae aisladas de chicha de jora del Valle del Mantaro (tesis de licenciatura). Universidad Nacional del Centro del Perú, Huancayo, Perú.

Granadillo, I.L., Tarantino, G., Hernández, R. \& Morán, H. (2014). Efecto de la temperatura y el pH en la fermentación del mosto de Agave cocui. Multiciencia, 14(4), 375-381.

Harmon, W. (2012). The complete idiot's guide to Fermenting foods ( $1^{\text {st. }}$ ed.). Estados Unidos de América: Alpha Books.

Ibañez, F.C. \& Barcina, Y. (2001). Análisis sensorial de alimentos: Métodos y aplicaciones. Barcelona, España: Springer-verlag Ibérica.

León, R. \&Zapata,A. (2008). Producción doméstica de la chicha. Revista 
Chicha Peruana, una bebida, una cultura, 1, $52-64$.

León, L. (2011). Proyecto deprefactibilidad para una planta productora de Chicha de Jora. Universidad Nacional de Trujillo, Perú.

Loviso, C.L. \& Dibkind, D. (2018). Síntesis y regulación de compuestos del aroma y el sabor derivados de la levadura en la cerveza: ésteres. Revista Argentina de Microbiología. https://doi. org/10.1016/j.ram.2017.11.006

Maupey, P., Grau, A., Barat, J. \& Albers, A. (2001). Introducción al secado de alimentos por aire caliente ( $1^{\text {a. }}$ ed.). España: Editorial Universidad Politécnica de Valencia.

Ministerio de Salud. (2009). Tablas Peruanas de composición de alimento ( $8^{\text {a }}$ ed.). Recuperado de http://www.ins.gob.pe/insvirtual/ images/otrpubs/pdf/Tabla\%20 de\%20Alimentos.pdf

Peninpede, M., Cohen, E., Schnoller, A., Blass, R., Pellon, H., Pettinato, H. \& Silvestre, A. (2003). HACCP, análisis de peligros y puntos críticos de control. Guía orientadora de productores, procesadores, y servicios de inspección. Revista Argentina de Humanidades y Ciencias Sociales, $14(1), 10-18$.

Pomasqui, J. (2012). Parámetros óptimos de la fermentación alcohólica para industrializar la chicha de jora en la procesadora de alimentos y bebidas Kutacachi Sara Mama (tesis liceciatura). Escuela Superior Politécnica de Chimborazo, Riobamba, Ecuador. Recuperado de http://dspace.espoch.edu.ec/ bitstream/123456789/2576/1/56 T00344.pdf

Rojas, B.S. (2013). Control de calidad y evaluación nutricional de las chichas (JorayMorada), elaboradas en la fundación Andinamarka, Calpi-Riobamba (tesis de licenciatura). Escuela Superior Politécnica de Chimborazo, Riobamba, Ecuador.

Saerens, S.M.G, Delvaux, F.R, Verstrepen, K.J. \& Thevelein, J.M. (2010). Production and biological function of volatile esters in Saccharomyces cerevisiae. Microbial Biotechnology, 3, $165-177$.

Salmón, J \& Ortiz-Julien, A. (2007). Mejora de la fermentación alcohólica en condiciones extremas. Artículo extraído de la ponencia presentada en el Congreso Microsafetywine, Vilafranca del Penedés, España, 20 y 21 Noviembre de 2007.

Sánchez, M., Muñoz, C., Di Carlo, J., Mayek, N. \& Hernández, J. (2010). Cambios Físico Químicos durante la germinación del maíz. Revista mexicana de Ciencias Agricolas, 1, 89 - 93.

Sulco, J.C. \& Alvarado, J.A. (2011). Estudio estadístico de pruebas sensoriales de harinas compuestas para panificación. Revista Boliviana de Química, 28(2), 79 -82 .

Teijón, J., Garrido, A., Blanco D., Villaverde, C., Mendoza, C. \& Ramirez, J. (2006). Fundamentos de Bioquimica Metabólica. España: Tébar S.L. 
Verstrepen, K.J, Derdelinnkcx, G., Dufour, J., Windericky, J., Thevelein, J.M. \& Pretorius, I.S. (2003). Flavor-active esters: Adding fruitiness to beer. Journal of Bioscience and Bioengineering, 96, 110-118.
Ward, O. (1991). Biotecnología de las Fermentaciones. Zaragoza, España: Editorial Acribia. 